\title{
Growth Differentiation Factor-15 as a Potent Predictor of Long-Term Mortality among Subjects with Osteoarthritis
}

\author{
Natalie Arnold ${ }^{1,2}$, Martin Rehm ${ }^{3}$, Gisela Büchele ${ }^{3}$, Raphael Simon Peter ${ }^{3}{ }^{\circledR}$, \\ Rolf Erwin Brenner ${ }^{4}$, Klaus-Peter Günther ${ }^{5}$, Hermann Brenner ${ }^{6,7,8}$ (), Wolfgang Koenig $3,9,10, *,+$ \\ and Dietrich Rothenbacher ${ }^{3,+}$ (D) \\ 1 Department of General and Interventional Cardiology, University Heart Centre Hamburg, \\ 20251 Hamburg, Germany; n.arnold@uke.de \\ 2 German Center for Cardiovascular Research (DZHK), partner site Hamburg/Kiel/Luebeck, \\ 20251 Hamburg, Germany \\ 3 Institute of Epidemiology and Medical Biometry, Ulm University, 89081 Ulm, Germany; \\ martin.rehm@uni-ulm.de (M.R.); Gisela.buechele@uni-ulm.de (G.B.); raphael.peter@uni-ulm.de (R.S.P.); \\ dietrich.rothenbacher@uni-ulm.de (D.R.) \\ 4 Division for Biochemistry of Joint and Connective Tissue Diseases, Department of Orthopedics, \\ Ulm University, 89081 Ulm, Germany; rolf.brenner@uni-ulm.de \\ 5 Technical University of Dresden, University Center of Orthopedic and Trauma Surgery, \\ 01069 Dresden, Germany; klaus-peter.guenther@uniklinikum-dresden.de \\ 6 Division of Clinical Epidemiology and Aging Research, German Cancer Research Center (DKFZ), \\ 69120 Heidelberg, Germany; h.brenner@Dkfz-Heidelberg.de \\ 7 Division of Preventive Oncology, German Cancer Research Center (DKFZ) and National Center for Tumor \\ Diseases (NCT), 69120 Heidelberg, Germany \\ 8 German Cancer Consortium (DKTK), German Cancer Research Center (DKFZ), 69120 Heidelberg, Germany \\ 9 Deutsches Herzzentrum München, Technische Universität München, 80636 Munich, Germany \\ 10 German Centre for Cardiovascular Research (DZHK), partner site Munich Heart Alliance, \\ 80333 Munich, Germany \\ * Correspondence: koenig@dhm.mhn.de; Tel.: + 49-89-1218-4073; Fax: +49-89-1218-2023 \\ + Equally contributing last authors.
}

Received: 29 July 2020; Accepted: 24 September 2020; Published: 26 September 2020

\begin{abstract}
Background: Subjects with osteoarthritis (OA) are at increased risk for cardiovascular $(\mathrm{CV})$ and all-cause mortality. Whether biomarkers improve outcome prediction in these patients remains to be elucidated. We investigated the association between growth differentiation factor 15 (GDF-15), a novel stress-responsive cytokine, and long-term all-cause mortality among OA patients. Methods: Within the Ulm Osteoarthritis Study, GDF-15 has been measured in the serum of 636 subjects, who underwent hip or knee arthroplasty between 1995 and 1996 (median age 65 years). Results: During a median follow-up of 19.7 years, a total of 402 deaths occurred. GDF-15 was inversely associated with walking distance. Compared to the bottom quartile (Q), subjects within the top quartile of GDF-15 demonstrated a 2.69-fold increased risk of dying (hazard ratio (HR) (95\% confidence interval (CI) 2.69 (1.82-3.96) adjusted for age, sex, BMI, smoking status, localization of OA, diabetes, maximum walking distance, total cholesterol, and cystatin $\mathrm{C}$. Further adjustment for NT-proBNP, troponin I, and hs-C-reactive protein did not change the results appreciably (HR $(95 \% \mathrm{CI})$ 1.56 (1.07-2.28); 1.75 (1.21-2.55); 2.32 (1.55-3.47) for Q2, Q3, and Q4 respectively, $p$ for trend < 0.001). Conclusions: In subjects with OA, GDF-15 represents a potent predictor of decreased survival over $>20$ years, independently of conventional CV risk factors, renal, cardiac, and inflammatory biomarkers as well as walking disability, previously associated with increased mortality and lower extremity OA.
\end{abstract}


Keywords: growth differentiation factor-15; total mortality; osteoarthritis; prognosis

\section{Introduction}

Osteoarthritis (OA) is a very common disorder and patients have an excess risk, especially for cardiovascular death [1]. This excess burden of cardiovascular disease (CVD) in patients with chronic connective tissue disorders [2,3] could not be solely explained by overrepresentation of conventional cardiovascular (CV) risk factors in this high-risk population. A highly active pro-inflammatory milieu with altered immune response might further predispose them to accelerated atherogenesis [3]. Thus, adequate risk assessment for future CVD events in these subjects is essential not only for estimating prognosis, but also for individualized patient care.

Although first attempts in this direction have already been undertaken, CVD risk prediction in these subjects is still challenging. Currently used risk stratification tools [4-8] regrettably are still not precise enough, leading to under- or overestimation of CVD risk in this patient population $[9,10]$. Even accounting for the pathophysiologic processes relevant for the underlying disease such as level of clinical disease activity (e.g., seropositivity for specific antibody and/or increased routine inflammatory parameters), extent of functional disability (e.g., joint erosions on X-ray), or disease duration and treatment modalities [11,12] did not lead to the desirable predictive accuracy for future CVD events [13]. More importantly, all these scores have been mainly applied in patients with rheumatic disorders, whereas data for other patient populations, such as with common age-related degenerative disease of the musculoskeletal system like OA, are still limited.

Another way to improve risk stratification beyond risk factors (traditional and non-traditional) is by using novel circulating biomarkers. Indeed, several studies have already related numerous proteins (inflammatory or cardiac) to adverse CVD outcome in patients with chronic degenerative/rheumatic disorders [14,15]. However, their incremental value beyond traditional CV risk factors or even other protein biomarkers has been evaluated to a lesser extent.

Recently, growth differentiation factor-15 (GDF-15), a member of the transforming growth factor-beta superfamily [16], has been identified as a novel and promising candidate across a spectrum of CVD ranging from acute ischemic events through chronic atherosclerotic disease to manifest heart failure $[17,18]$. Moreover, elevated GDF-15 has been recognized as a powerful predictor of bleeding in subjects receiving dual antiplatelet therapy or in those on anticoagulant therapy $[19,20]$. In contrast to other established CVD biomarkers, GDF-15 is also strongly related to numerous non-cardiovascular conditions such as kidney diseases, diabetes mellitus, neoplasia and cancer-induced anorexia, or rheumatoid arthritis $[17,18]$. Even in young subjects without overt CVD, GDF-15 might represent an early indicator of CV risk development [21]. Finally, its strong association with all-cause mortality [22-31] might also imply a crucial role of GDF-15 in biological processes associated with ageing. However, data from patient populations with osteoarthritis are rare yet.

Considering the involvement of GDF-15 in controlling various physiological and pathological conditions, we thought to investigate whether GDF-15 is able to predict increased long-term risk of all-cause mortality beyond traditional risk factors and biochemical risk markers in a high-risk population for CVD, like patients with OA.

\section{Materials and Methods}

\subsection{Study Design and Population}

The Ulm Osteoarthritis Study represents a multicenter prospective cohort study of OA patients with unilateral total hip or knee replacement between January 1995 and December 1996. Details of the study design have been reported elsewhere [32]. Briefly, at baseline, 809 consecutive patients, who underwent unilateral total hip or knee arthroplasty due to advanced OA, were eligible for the 
study. Exclusion criteria were age $>75$ years, malignancies, inflammatory diseases, corticosteroid medication, or previous hip or knee joint replacement. Participation was voluntary and written informed consent was obtained from each subject upon entry into the study. The study protocol and study documents were approved by the Ethics Committee of Ulm University (No. 164/14) and were performed according to the principles of Good Clinical Practice and the Declaration of Helsinki.

For the present report, 173 participants with missing information on biomarker concentrations at baseline were excluded. In total, 636 subjects with all information on variables of interest were considered for the analyses. All-cause mortality was used as the primary outcome of the study. Death was ascertained by obtaining the survival status via the respective residents' registration office. Mortality was assessed during follow-up (FU) at 6, 12, and 60 months after joint replacement and again, in the years 2014, 2015, and 2019 (last update was 11 June 2019). Information on living status could be traced for $98.4 \%$ of participants.

\subsection{Data Collection}

All subjects underwent a baseline examination in a standardized manner according to the study protocol in Ulm, Augsburg, or Stuttgart (three cities in the South of Germany). Data were collected by standardized questionnaires in personal interviews and included demographic and lifestyle-related data (e.g., age, sex, weight, height, smoking status) and information on self-reported medical history (e.g., diabetes mellitus, hypertension, myocardial infarction, and heart failure). Finally, OA-related variables such as an assessment of maximum walking distance (in six categories) as well as the functionality subscale of the Western Ontario and McMaster University Osteoarthritis Index (WOMAC) (score range 0-68) used to specifically describe function in the operated joint at baseline assessment (preoperative) and the pain subscale of the WOMAC (pain score 0-20) as a measure of paint intensity were also applied.

\subsection{Laboratory Methods}

Venous blood was drawn preoperatively under standardized conditions from all study participants after an overnight fasting period. All samples were stored at $-80^{\circ} \mathrm{C}$ until further analysis. No samples were inadvertently thawed during storage. All routine laboratory parameters were determined on the day of sampling by standard methods in the central laboratory of participating centers.

The following biomarkers were measured in frozen serum samples in 2018: serum cystatin C was measured by immunonephelometry on a BNA II (Behring Co. Marburg, Germany) with an inter-assay coefficient of variation $(\mathrm{CV})$ between $1.7 \%$ and $2.7 \%$. High sensitivity C-reactive protein (hs-CRP) was measured using latex-enhanced nephelometry (NA-latex CRP, Behring Co., Marburg, Germany), calibrated with the WHO reference standard 85/506 (inter-assay CVs between $2.1 \%$ and $3.2 \%$ ). N-terminal pro-B-type natriuretic peptide (NT-proBNP) concentrations were determined on an Elecsys (Roche Diagnostics, Penzberg, Germany) with a limit of detection (LoD) of $5.0 \mathrm{ng} / \mathrm{L}$ and an inter-assay CV $<5 \%$. Concentrations of hs-cardiac troponin I (hs-cTnI) were measured on an Architect STAT (Abbott Diagnostics, Wiesbaden, Germany) with a LoD of $2.0 \mathrm{ng} / \mathrm{L}$ and inter-assay CVs between $3.8 \%, 3.9 \%$, and $5.6 \%$ at three different concentrations.

Baseline sCOMP was analyzed using a commercial sandwich ELISA against human COMP (BioVendor, Heidelberg, Germany; LOD $<0.4 \mathrm{ng} / \mathrm{mL}$ ). The assay was run according to the manufacturer's instructions, using a sample dilution of 1:50. Both inter- (CV 15.6\%) and intra-assay variations (CV 2.8\%) were assessed as quality control. The absorbance was detected at $450 \mathrm{~nm}$ and a reference wavelength at $630 \mathrm{~nm}$ by using the multimode microplate reader Infinite M200 Pro (Tecan Austria GmbH, Groedig, Austria).

Finally, GDF-15 serum concentration was measured by Electrochemiluminescence Immunoassay (ECLIA, Cobas Elecsys 411, Roche Diagnostics, Penzberg, Germany) with a measuring range of $27.6-12,700 \mathrm{ng} / \mathrm{L}$, a LoD of $10 \mathrm{ng} / \mathrm{L}$, and inter-assay CVs of $4.6 \%$ and $4.9 \%$ at two different concentrations. All analyses were run in a blinded fashion. 


\subsection{Statistical Analysis}

Baseline demographic, clinical, and laboratory characteristics were compared in a descriptive way. For the present analysis, GDF-15 concentrations were categorized into quartiles $(\mathrm{Q})$ with a cut-point of $<780,780-<1006,1006-1279$, and $>1279 \mathrm{ng} / \mathrm{L}$, respectively. Continuous variables were reported as medians, with their interquartile ranges (IQR, 25th and 75th percentile); categorical variables were presented as absolute frequencies (percentage and number). Measured biomarkers were natural log-transformed prior to the analysis, where appropriate.

Partial Spearman correlation coefficients, adjusted for age and sex, were calculated to describe the correlation between GDF-15 and numerous variables of interest.

Multivariable Cox proportional hazard analysis with various degrees of adjustment was carried out to investigate the predictive value of GDF-15 on long-term all-cause mortality. Concentrations of GDF-15 were divided into quartiles of their distribution (as outlined above), with quartile one as the reference quartile, or were used continuously (per unit increase after log-transformation). A basic model was adjusted for age (in years) and sex (male, female). A second model further included body mass index (BMI) (continuous $\mathrm{kg} / \mathrm{m}^{2}$ ), smoking status (never, former, current), localization of the OA (hip, knee), history of diabetes mellitus (yes/no), serum total cholesterol (continuous), maximum walking distance (in categories), and continuous log-transformed cystatin C concentrations. The final model was additionally adjusted for continuous log-transformed concentrations of hs-CRP, hs-cTnI, and NT-proBNP. Results are reported as Hazard Ratios (HR) with their $95 \%$ confidence intervals (CIs). In addition, a dose-response relationship between GDF-15 and outcome was plotted with restricted cubic splines using four knots and the median concentration in the lowest category as the reference. The Cox proportional regression analysis was conducted for the whole follow-up period, as well as repeated under the same conditions using a differential FU time (i.e., after 5, 10, 20, and 24 years of FU).

Finally, to investigate whether GDF-15 might improve risk assessment beyond the established risk predictors in OA patients, several measures of model accuracy were performed. First, to assess discrimination of events, areas under the receiver-operating characteristic curve (AUC) were calculated for models without ("basic" model) and with biomarkers (adding each of the biomarkers individually: hs-CRP, hs-cTnI, NT-proBNP, and GDF-15). The first, "basic" model contained age, sex, BMI, smoking status, localization of $\mathrm{OA}$, diabetes mellitus, cholesterol, and log-transformed concentration of cystatin C. A second, "full" model contained in addition to the above mentioned variables and in addition hs-CRP, hs-cTnI, and NT-proBNP as reference models and then, GDF-15 was added. Furthermore, the net reclassification improvement (NRI) in cases and non-cases calculated by the Kaplan-Meier estimator was used to examine the predictive value of studied biomarkers according to the $1 \%, 5 \%$, and $10 \%$ risk strata of the predicted probability for death after ten years [33]. The bootstrap method was used to create $95 \%$ CI for the NRI estimates [34]. Statistical analysis was performed using SAS version 9.4 (SAS Institute Inc., Cary, NC, USA) and R version 3.5.1 (The R Foundation for Statistical Computing, Vienna, Austria).

\section{Results}

In the total population of 636 participants with OA, the median (IQR) GDF-15 concentration at baseline was $1005.5 \mathrm{ng} / \mathrm{L}$ (780.0-1280.5 ng/L). Baseline demographic, clinical, and laboratory characteristics of the study participants in quartiles of the GDF-15 distribution are summarized in Table 1. 
Table 1. Demographic, clinical, and laboratory characteristics of the study participants at baseline by quartiles of GDF-15.

\begin{tabular}{|c|c|c|c|c|c|c|}
\hline & & $\begin{array}{c}\mathrm{Q1} \\
<780.0 \mathrm{ng} / \mathrm{L}\end{array}$ & $\begin{array}{c}\mathrm{Q} 2 \\
780.0-<1006.0 \mathrm{ng} / \mathrm{L}\end{array}$ & $\begin{array}{c}\mathrm{Q3} \\
1006.0-1279.0 \mathrm{ng} / \mathrm{L}\end{array}$ & $\begin{array}{c}Q 4 \\
>1279.0 \mathrm{ng} / \mathrm{L}\end{array}$ & Total \\
\hline$n$ & & 158 & 160 & 159 & 159 & 636 \\
\hline Age, years & & $58.0(53.0 / 64.0)$ & $65.0(60.0 / 69.0)$ & $67.0(61.0 / 71.0)$ & $70.0(65.0 / 72.0)$ & $65.0(58.0 / 70.0)$ \\
\hline \multirow[t]{2}{*}{ Sex, \% $(n)$} & Male & $46.8(74)$ & $32.5(52)$ & $35.8(57)$ & $27.7(44)$ & $35.7(227)$ \\
\hline & Female & $53.2(84)$ & $67.5(108)$ & $64.2(102)$ & $72.3(115)$ & $64.3(409)$ \\
\hline Body Mass Index, kg/m² & & $27.3(25.0 / 29.8)$ & $27.8(25.3 / 30.1)$ & $27.8(24.8 / 30.9)$ & $28.7(25.9 / 32.4)$ & $27.8(25.5 / 30.9)$ \\
\hline \multirow[t]{2}{*}{ Localization of OA, \% $(n)$} & Hip & $63.9(101)$ & $56.3(90)$ & $51.6(82)$ & $38.4(61)$ & $52.5(334)$ \\
\hline & Knee & $36.1(57)$ & $43.8(70)$ & $48.4(77)$ & $61.6(98)$ & $47.5(302)$ \\
\hline \multirow[t]{3}{*}{ Smoking status, $\%(n)$} & Never & $55.7(88)$ & $58.8(94)$ & $59.1(94)$ & $59.1(94)$ & $58.2(370)$ \\
\hline & Former & $33.5(53)$ & $28.8(46)$ & $27.7(44)$ & $30.8(49)$ & $30.2(192)$ \\
\hline & Current & $10.8(17)$ & $12.5(20)$ & $13.2(21)$ & $10.1(16)$ & $11.6(74)$ \\
\hline \multicolumn{2}{|c|}{ History of diabetes mellitus, $\%(n)$} & $3.2(5)$ & $6.3(10)$ & $11.9(19)$ & $13.8(22)$ & $8.8(56)$ \\
\hline \multicolumn{2}{|c|}{ History of hypertension, \% $(n)$} & $41.1(65)$ & $45.6(73)$ & $51.6(82)$ & $67.3(107)$ & $51.4(327)$ \\
\hline \multicolumn{2}{|c|}{ History of myocardial infarction, \% ( $n$ ) } & $3.8(6)$ & $1.3(2)$ & $4.4(7)$ & $5.7(9)$ & $3.8(24)$ \\
\hline \multicolumn{2}{|c|}{ History of heart failure, $\%(n)$} & $6.3(10)$ & $16.9(27)$ & $17.0(27)$ & $33.3(53)$ & $18.4(117)$ \\
\hline \multicolumn{2}{|c|}{ Total cholesterol, $\mathrm{mmol} / \mathrm{L}$} & $5.6(5.2 / 6.3)$ & $5.7(5.0 / 6.4)$ & $5.8(5.0 / 6.3)$ & $5.8(5.1 / 6.4)$ & $5.7(5.1 / 6.4)$ \\
\hline \multicolumn{2}{|l|}{ Triglyceride, mmol/L } & $1.4(1.0 / 2.0)$ & $1.4(1.0 / 2.1)$ & $1.6(1.0 / 2.3)$ & $1.5(1.1 / 2.2)$ & $1.5(1.0 / 2.1)$ \\
\hline \multicolumn{2}{|l|}{ Cystatin C, mg/L } & $0.8(0.7 / 0.9)$ & $0.8(0.8 / 0.9)$ & $0.9(0.8 / 1.0)$ & $1.0(0.9 / 1.2)$ & $0.9(0.8 / 1.0)$ \\
\hline \multicolumn{2}{|l|}{ hs-CRP, mg/L } & $2.0(1.0 / 3.8)$ & $2.5(1.1 / 4.9)$ & $2.5(1.4 / 6.2)$ & $3.0(1.8 / 6.3)$ & $2.5(1.3 / 5.0)$ \\
\hline \multicolumn{2}{|l|}{ hs-cTnI, ng/L } & $3.1(2.3 / 4.7)$ & $3.7(2.7 / 5.3)$ & $3.9(2.9 / 5.5)$ & $5.3(3.8 / 7.6)$ & $3.9(2.8 / 5.7)$ \\
\hline \multicolumn{2}{|l|}{ NT-proBNP, ng/L } & $67.7(32.7 / 118.3)$ & $93.5(49.9 / 167.5)$ & $100.1(62.0 / 162.3)$ & $137.8(84.6 / 269.1)$ & $99.1(52.2 / 83.9)$ \\
\hline \multicolumn{2}{|l|}{ sCOMP ng/mL } & $751.0(545.3 / 980.8)$ & $783.2(586.8 / 1064.8)$ & $826.4(626.6 / 1138.6)$ & $831.4(626.2 / 1080.6)$ & $797.1(598.6 / 1044.5)$ \\
\hline \multicolumn{2}{|l|}{ WOMAC pain score } & $12.0(9.0 / 14.0)$ & $11.5(9.0 / 13.0)$ & $12.0(10.0 / 15.0)$ & $13.0(10.0 / 15.0)$ & $12.0(10.0 / 14.0)$ \\
\hline \multicolumn{2}{|l|}{ WOMAC function score } & $39.5(31.0 / 46.0)$ & $39.5(32.0 / 46.0)$ & $39.0(31.0 / 46.0)$ & $40.0(33.0 / 48.0)$ & $39.5(32.0 / 46.0)$ \\
\hline Maximum walking distance & $>1000 \mathrm{~m}$, but limited & $31.8 *(55)$ & $24.9 *(43)$ & $21.4 *(37)$ & $22.0 *(38)$ & $27.2(173)$ \\
\hline \multicolumn{2}{|c|}{$\sim 1000 \mathrm{~m}$ or $\sim 15 \mathrm{~min}$} & $30.9 *(29)$ & $19.2 *(18)$ & $25.5 *(24)$ & $24.5 *(23)$ & $14.8(94)$ \\
\hline \multicolumn{2}{|c|}{$500-900 \mathrm{~m}$ or $\sim 8-15 \mathrm{~min}$} & $26.2 *(27)$ & $27.2 *(28)$ & $22.3 *(23)$ & $24.3 *(25)$ & $16.2(103)$ \\
\hline & $300-500 \mathrm{~m}$ & $18.0 *(15)$ & $25.3 *(21)$ & $26.5 *(22)$ & $30.1 *(25)$ & $13.1(83)$ \\
\hline & $100-300 \mathrm{~m}$ & $19.6 *(22)$ & $27.7 *(31)$ & $32.1 *(36)$ & $20.5 *(23)$ & $17.6(112)$ \\
\hline & $<100 \mathrm{~m}$ & $14.1 *(10)$ & $26.8 *(19)$ & $23.9 *(17)$ & $35.2 *(25)$ & $11.2(71)$ \\
\hline
\end{tabular}

Data are expressed as a median (25th/75th percentile) or in column $\%(n)$ if not indicated otherwise ${ }^{*}$ ) as row \%. GDF-15-growth differentiation factor-15; Q-quartile; OA—osteoarthritis; hs-CRP-high-sensitive C-reactive protein; hs-cTnI-high-sensitive troponin I; NT-proBNP-N-terminal pro-B-type natriuretic peptide; sCOMP-serum cartilage oligomeric matrix protein; WOMAC —Western Ontario and McMaster University Osteoarthritis Index; $\mathrm{m}$-meters; min—minutes. 
Subjects with higher GDF-15 concentrations were older and more likely to be females. Furthermore, the prevalence of diabetes mellitus and hypertension increased markedly with increasing GDF-15 concentrations. In addition, BMI, frequency of previous myocardial infarction, and percentage of never smokers tended to be higher in higher GDF-15 quartiles. No clear association was revealed throughout the GDF-15 quartiles with the presence of former or current smoking. Interestingly, the proportion of subjects who underwent total knee replacement was higher in the upper GDF-15 quartile concentrations compared to patients with hip OA. It should be noted here, however, that subjects with knee OA tended to be older compared to subjects with hip OA (median (IQR: 67 (64-71) years versus 62 (55-69) years).

With regard to the laboratory parameters, subjects with higher GDF-15 concentrations had also higher concentrations of most investigated analytes (Table 1), with the only exception being for triglycerides.

In addition, an inverse relationship has been observed between maximum walking distance and GDF-15 concentrations, with the higher values of GDF-15 associated with shorter walking distance.

Next, we calculated age- and sex-adjusted partial Spearmen rank correlations coefficients between GDF-15 and cardiac, lipid, and renal parameters (Table 2).

Table 2. Correlation between GDF-15 and several biochemical parameters, adjusted for age and sex.

\begin{tabular}{ccc}
\hline & Partial Spearman Rank Correlation Coefficients (Rho) & $p$-Values \\
\hline Total cholesterol & -0.026 & 0.527 \\
Triglyceride & 0.093 & 0.036 \\
Cystatin C & 0.371 & $<0.001$ \\
hs-CRP & 0.173 & $<0.001$ \\
hs-cTnI & 0.230 & $<0.001$ \\
NT-proBNP & 0.127 & 0.001 \\
sCOMP & 0.078 & 0.050 \\
\hline
\end{tabular}

GDF-15—growth differentiation factor-15; Q-quantile; OA—osteoarthritis; hs-CRP—high-sensitive C-reactive protein; hs-cTnI-high-sensitive troponin I; NT-proBNP-N-terminal pro-B-type natriuretic peptide—sCOMP—serum cartilage oligomeric matrix protein.

The strongest correlation was found for GDF-15 and cystatin C with a Rho of $0.371(p<0.001)$, followed by both troponins ( $R h o=0.230 ; p<0.001)$ and hs-CRP (Rho $=0.173 ; p<0.001)$. Surprisingly, NT-proBNP and sCOMP were only modestly correlated to GDF-15 (Rho $=0.127$ and Rho $=0.078$, respectively) (Table 2).

Finally, we were interested in the prognostic evaluation of GDF-15 for all-cause mortality. The association between concentrations of GDF-15 at baseline and all-cause mortality over a median FU of 19.7 years was assessed both in quartiles of the GDF-15 distribution as well as continuously (per log unit (U) increase in GDF-15 concentration) (Table 3).

During FU, a total of 402 (63.2\%) deaths occurred. Deceased subjects demonstrated markedly higher median GDF-15 concentrations at baseline than non-cases (1120.5 vs. $796.7 \mathrm{ng} / \mathrm{L}$, respectively, data not shown). Total mortality rate was found to be 36.4 deaths per 1000 person-years and increased from 14.7 per 1000 person-years in quartile 1 to a mortality rate of 61.7 deaths per 1000 person-years in the quartile 4 (Table 3). 
Table 3. Association between GDF-15 concentration at baseline and risk of long-term all-cause mortality in subjects with osteoarthritis.

\begin{tabular}{|c|c|c|c|c|c|c|}
\hline & & Events/ $\mathbf{N}$ & Rate per $1000 p$-yr & Model 1HR (95\% CI) & Model 2HR (95\% CI) & Model 3HR (95\% CI) \\
\hline \multirow[t]{6}{*}{ GDF-15 } & Quartile $1(<780.0$ ng/L) & $48 / 158$ & 14.7 & 1.00 & 1.00 & 1.00 \\
\hline & Quartile 2 (780.0-1006.0 ng/L) & $100 / 160$ & 35.0 & $1.85(1.30-2.63)$ & $1.64(1.12-2.38)$ & $1.56(1.07-2.28)$ \\
\hline & Quartile 3 (1006.0-1279.0 ng/L) & $118 / 159$ & 43.3 & $2.06(1.45-2.93)$ & $1.84(1.27-2.66)$ & $1.75(1.21-2.55)$ \\
\hline & Quartile $4(>1279.0 \mathrm{ng} / \mathrm{L})$ & $136 / 159$ & 61.7 & $2.93(2.05-4.18)$ & $2.69(1.82-3.96)$ & $2.32(1.55-3.47)$ \\
\hline & $p$ for trend & & & $<0.001$ & $<0.001$ & $<0.001$ \\
\hline & Per log unit increase & $402 / 636$ & & $2.41(1.88-3.10)$ & $2.34(1.75-3.14)$ & $2.12(1.56-2.88)$ \\
\hline
\end{tabular}

Results from multivariable adjusted Cox proportional hazards model with all-cause mortality as dependent variable. Median (25th/75th percentile) follow-up time is 19.7 (12.6/22.9) years Model 1 was adjusted for age and sex. Model 2 was adjusted for age, sex, BMI, smoking status, localization of OA, diabetes, maximum walking distance, total cholesterol, and log-transformed concentration of cystatin C. Model 3 was additionally adjusted for log-transformed concentrations hs-CRP, hs-cTnI, and NT-proBNP. N-number of observed subjects; $p$-yr-person-years; HR - hazard ratio; CI—confidence interval; GDF-15—growth differentiation factor-15; OA for osteoarthritis; hs-CRP-high-sensitive C-reactive protein; hs-cTnI-high-sensitive troponin I; NT-proBNP-N-terminal pro-B-type natriuretic peptide. 
The results of multivariable Cox proportional hazard analyses are presented in Table 3. After adjustment for age and sex, subjects within Q2, Q3, and Q4 of the GDF-15 distribution demonstrated a 2- to 3-fold increased risk of dying from all-causes, compared to those within the bottom quartile (Q1) (HR (95\% CI) 1.85 (95\% CI 1.30-2.63), 2.06 (95\% CI,1.45-2.93), and 2.93 (95\% CI, 2.05-4.18), respectively; $p$ for trend $<0.001)$. Further adjustment for well-established confounders such as BMI, smoking status, localization of $\mathrm{OA}$, diabetes, maximum walking distance, total cholesterol, and cystatin $\mathrm{C}$ in model 2 led to only minimal reduction in estimates, with a HR of 1.64 (95\% CI 1.12-2.38) for Q2 and a HR of 1.84 (95\% CI 1.27-2.66) for Q3, and a HR of 2.69 (95\% CI 1.82-3.96) for Q4, respectively. Most importantly, additional simultaneous adjustment for NT-proBNP, hs-cTnI, and hs-CRP did not change the results appreciably (HR (95\%CI) 1.56 (95\%CI 1.07-2.28), $1.75(95 \% \mathrm{CI} 1.21-2.55)$ and 2.32 (95\%CI 1.55-3.47) for Q2, Q3, and Q4 versus Q1 respectively, $p$ for trend <0.0001). Almost identical results in the different models were seen if GDF-15 concentrations were taken in the analysis as a continuous variable with a HR of 2.12 (95\% CI 1.56-2.88) in the fully adjusted model.

Proportional hazard estimates for all-cause mortality across all ranges of GDF-15 concentrations based on a fully adjusted multivariable model (Model 3 in Table 3) are graphically presented in Figure 1.

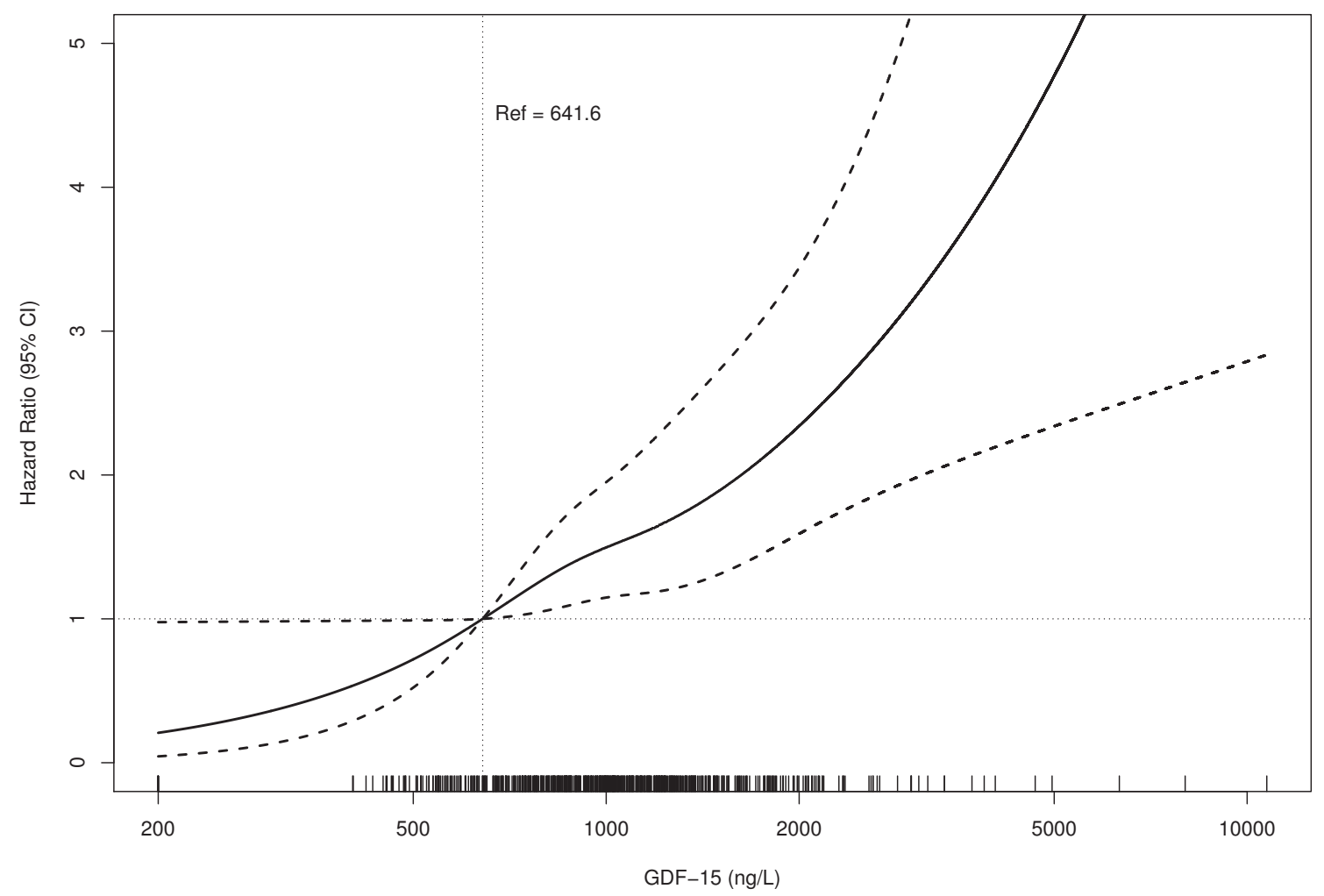

Figure 1. Relationship between GDF-15 Concentration and Mortality.(HR bold line, 95\% CI dotted lines).Adjusted for age, sex, BMI, smoking status, localization of OA, diabetes, maximum walking distance, total cholesterol, and log-transformed concentrations of cystatin C, hs-CRP, hs-cTnI, and NT-proBNP (Model 3 from Cox proportional hazards analysis). BMI-body mass index; $\mathrm{OA}$, osteoarthritis; hs-CRP_high-sensitive C-reactive protein; hs-cTnI-high-sensitive troponin I; NT-proBNP-N-terminal pro-B-type natriuretic peptide; GDF-15-growth differentiation factor-15.

In addition, we repeated the Cox proportional analysis under the same levels of adjustments, truncating follow-up at 5-, 10-, and 20-years and saw no substantial changes in HRs with increasing FU period, documenting the robustness of the GDF-15 risk estimates over follow-up time (Figure S1 Supplemental Materials).

To investigate whether GDF-15 might improve risk assessment beyond established risk predictors, several measures of model accuracy were calculated, as shown in Table 4. 
Table 4. Measures of model discrimination and reclassification for all-cause mortality in subjects with osteoarthritis.

\begin{tabular}{cccc}
\hline & AUC (95\% CI) & NRI $_{\mathbf{e}}$ (95\% CI) & NRI $_{\text {ne }}$ (95\% CI) \\
\hline Basic model $^{\text {a }}$ & $0.73(0.71 / 0.76)$ & & \\
Basic model + ln(hs-CRP) & $0.73(0.71 / 0.76)$ & $-0.01(-0.06 / 0.03)$ & $0.01(-0.01 / 0.06)$ \\
Basic model + ln(hs-cTnI) & $0.74(0.72 / 0.77)$ & $-0.01(-0.08 / 0.06)$ & $0.06(0.02 / 0.14)$ \\
Basic model + ln(NT-proBNP) & $0.74(0.72 / 0.77)$ & $0.05(-0.05 / 0.09)$ & $0.09(0.01 / 0.17)$ \\
Basic model + ln(GDF-15) & $0.74(0.72 / 0.77)$ & $0.02(-0.06 / 0.10)$ & $0.06(0.00 / 0.13)$ \\
Full model ${ }^{\text {b }}$ & $0.75(0.72 / 0.77)$ & & \\
Full model + ln(GDF-15) & $0.76(0.73 / 0.78)$ & $-0.01(-0.06 / 0.07)$ & $0.04(-0.01 / 0.09)$ \\
\hline
\end{tabular}

\begin{abstract}
a Basic model (Model 2 from Cox proportional hazards analysis) was adjusted for age, sex, BMI, smoking status, localization of OA, diabetes, maximum walking distance, cholesterol, and log-transformed concentration of cystatin C. ${ }^{b}$ Full model (Model 3 from Cox proportional hazards analysis) was adjusted for age, sex, BMI, smoking status, localization of $\mathrm{OA}$, diabetes, maximum walking distance, total cholesterol, and log-transformed concentrations of cystatin C, hs-CRP, hs-cTnI, and NT-proBNP. AUC-areas under the receiver-operating characteristic curve; $\mathrm{NRI}_{e}$ - event net reclassification index; $\mathrm{NRI}_{n e}$-non-event net reclassification index; hs-CRP—high-sensitive C-reactive protein; hs-cTnI-high-sensitive troponin I; NT-proBNP-N-terminal pro-B-type natriuretic peptide; GDF-15-growth differentiation factor-15.
\end{abstract}

The addition of log GDF-15 or other investigated biomarkers to the basic model led to only minimal improvement in the AUC, thereby indicating an almost negligible incremental value of these parameters for prediction of all-cause mortality. However, increase from 0.73 (95\% CI, 0.71-0.76) to 0.76 (95\% CI, 0.73-0.78) was seen when all biomarkers were considered together. Reclassification analysis in the deceased further showed only as slightly different from zero NRI (event NRI), whereas non-event NRI for GDF-15 beyond factors included in the basic model was found to be $0.06(95 \% \mathrm{CI} 0.00-0.13)$, indicating a correct downward reclassification of non-events. Interestingly, further reclassification (downwards) was seen if GDF-15 was evaluated additionally in the second, "full" model including other biomarkers ( $\mathrm{NRI}_{\text {ne }} 0.04$ (95\%CI -0.01-0.09)); however, the 95\% CI included the null-effect value.

\title{
4. Discussion
}

To the best of our knowledge, the present analysis represents the first prospective study investigating the prognostic value of the novel promising biomarker GDF-15 for all-cause mortality in subjects with OA considering a large number of established and new cardiovascular biomarkers. Our major finding showed higher GDF-15 concentration to predict worse survival both short- and long-term over $>20$ years in this high-risk population. Importantly, the observed association between increased GDF-15 concentrations and a higher risk of dying from all causes was independent not only of well-known potential confounders like traditional CVD risk factors or markers of renal dysfunction. Even after simultaneous adjustment for walking disability as well as for established strong predictors of future CVD events like hs-CRP, hs-TnI, and NT-proBNP, GDF-15 remained a potent risk indicator of all-cause death with a 2-times-higher rate of those in the top compared to the lowest GDF-15 quartile. Thus, in subjects with OA, GDF-15 provides additional prognostic information on all-cause mortality, which is not captured by conventional risk factors, "maximum walking distance" as a measure of gait disability, or other well-established biomarkers and may play a role in early risk stratification measures.

The independent association between elevated GDF-15 concentrations and short- or long-term total mortality has been already conclusively demonstrated among subjects from the general population as well as in various patient cohorts $[17,18,22-31]$ and some of the studies had used the same analysis platform than our study $[25,26,29]$. Interestingly, several studies $[23,30,31]$ have demonstrated that GDF-15 predicted all-cause mortality independently of and more accurately than other biomarkers like NT-proBNP, hs-CRP, or hs-cTn. Furthermore, in the general population [23,24], GDF-15 was the only biomarker additionally predictive for non-CVD and cancer mortality. Robust relations of GDF-15 to non-cardiovascular events, which was not seen for NT-proBNP, troponins, and hs-CRP, distinguishes it 
from other abovementioned CVD risk markers and suggests additional regulatory mechanisms for GDF-15 expression.

Indeed, GDF-15 represents a stress-responsive cytokine with multiple mechanisms of action [16]. While weakly expressed under physiological conditions, GDF-15 is highly secreted as a stress response to inflammation, oxidative stress, hypoxia, telomere erosion, and oncogene activation. GDF-15 has also been considered as a marker of frailty due to its association with mitochondrial dysfunction [35]. Nonetheless, biological processes that could explain a link between increased GDF-15 concentrations and various cardiac and non-cardiac outcomes are still not entirely elucidated. One might speculate that GDF-15 could play a dual (deleterious and protective) role in a variety of settings, as it e.g., has been recently proposed for cancer [16]. Premature or biological immunosenescence with accompanying "inflammaging" [36] might represent other possible mechanisms, relating GDF-15 to multiple age-related phenotypes and pathologies like OA, cancer, neurodegenerative disorders, frailty, or CVD. Two recently published proteome-based analyses [37,38] provided the first evidence on such associations. In the first study by Tanaka et al. [37], GDF-15 was found to be one of the strongest biomarkers associated with chronological age among 1301 tested proteins. Within the other analysis [38], GDF-15 has been demonstrated as a robust marker of senescence-associated secretory phenotypes, being simultaneously identified as a protein, highly secreted following all senescence-inducing stimuli, and as a biomarker, being highly related to aging.

Another unexpected finding from the present analysis was related to the fact that among OA subjects, GDF-15 was independently predictive for total mortality already at concentrations between 780 and $1006 \mathrm{ng} / \mathrm{L}$ (i.e., at Q2 of GDF-15 distribution with a median age of 65.0 years), where a HR of 1.56 (95\% CI 1.07-2.28) was observed after adjustment for potential confounders, including other risk biomarkers. Several studies $[26,28]$ have proposed a cut-off $>1800 \mathrm{ng} / \mathrm{L}$ to identify high-risk subjects based on elevated concentrations of GDF-15. Although not directly comparable with the current analysis, data from the general population [23] or from several patient cohorts [26] demonstrated any meaningful association with all-cause mortality at much higher concentrations. Interestingly, the 50th percentile of GDF-15 concentration within the general population was found to be $945 \mathrm{ng} / \mathrm{L}$ at an age of 65 years [39]. Transferring these reference values to our results, one might conclude that subjects with OA have a 56\% increased risk of dying, even being within the age-related reference limit of GDF-15.

Our study has several strengths that need to be mentioned. First, the present analysis was based on a well-characterized patient cohort including more than 600 subjects. Simultaneous assessment of novel and established biomarkers of different key pathways as well as a long FU period represent further strengths. Despite the intriguing results, several limitations of the current study also merit consideration. One of them is generalizability, since only Central Europeans aged 28 to 75 years were investigated. Furthermore, only subjects with advanced hip or knee OA, which required unilateral joint replacement, were included, which represent a selective population. No detailed information on long-term stability of the measured biomarkers in frozen samples was available, so we could not completely exclude that longer FU might influence the association between the risk marker and the disease outcome. However, in that case, we would expect rather a weakening of the associations found. Moreover, in another study, evaluating the 8-year stability of e.g., NT-proBNP serum samples stored at $-80^{\circ} \mathrm{C}$ with a maximum of one defrost cycle, we found an estimated recovery between $89.5 \%$ and $103 \%$ [40]. In addition, the platform used to measure GDF-15 in each study has to be considered. Finally, since all-cause mortality was the sole outcome in the present study, a predictive value of GDF-15 on CVD and non-CVD mortality has not been examined.

This is the first prospective study in patients with OA, demonstrating a strong prognostic value of elevated GDF-15 on decreased survival over $>20$ years. More importantly, the observed association was not affected by conventional CV risk factors as well as numerous well-established renal, cardiac, and inflammatory biomarkers. Although the results should preferably be verified in other cohorts, our findings suggest GDF-15 as a robust risk marker with a predictive value persisting even decades later. Thus, we believe that GDF-15, being not only strongly involved in biological processes associated with 
ageing but rather indicating an overall response to various stressors, might be a useful biomarker for future risk stratification or targeting preventive measures in such high-risk populations like subjects with OA.

Supplementary Materials: The following are available online at http:/www.mdpi.com/2077-0383/9/10/3107/s1, Figure S1: Cox Proportional Regression Analysis for Total Mortality Using Different Follow-up Periods.

Author Contributions: Writing—original draft-lead, N.A.; formal analysis-lead, M.R.; formal analysis-supporting G.B.; formal analysis-supporting, R.S.P.; conceptualization-equal, data curation-equal, funding acquisition-equal, R.E.B.; conceptualization-equal, data curation-equal, K.-P.G.; funding acquisition-equal, methodology-equal, supervision-equal, H.B.; conceptualization-equal, funding acquisition-equal, supervision-equal, W.K.; conceptualization-equal, funding acquisition-equal, supervision-equal, D.R. All authors have read and agreed to the published version of the manuscript.

Funding: This work was supported by a grant from the German Federal Ministry for Research and Technology (BMFT No. 01 EF940/6). Roche Diagnostics, Penzberg, Germany, kindly provided reagents for GDF-15 for free. The last follow-up was supported by the Deutsche Arthrosehilfe e.V.

Acknowledgments: We are indebted to all members of the Ulm Osteoarthritis Study for being involved in the planning and conducting of this study. In addition, we express our appreciation to all study participants. The authors would like to thank Gerlinde Trischler for her expert technical support.

Conflicts of Interest: N.A., M.R., G.B., R.S.P., and H.B. have no conflict of interest to declare. K.-P.G. reports research grants from Zimmer, Link, and Aesculap outside the submitted work. R.E.B. reports grants from Deutsche Arthrosehilfe e.V., during the conduct of the study; and grants from the German Research Foundation and grants from the German Society for Orthopedics and Orthopedic Surgery outside the submitted work. D.R. reports grants from the German Federal Ministry for Research and Technology and grants from Deutsche Arthrosehilfe e.V during the conduct of the study; W.K. reports personal fees from AstraZeneca, personal fees from Novartis, personal fees from Pfizer, personal fees from The Medicines Company, personal fees from DalCor, personal fees from Kowa, personal fees from Amgen, personal fees from Corvidia, personal fees from Daiichi-Sankyo, personal fees from Berlin-Chemie, personal fees from Sanofi, personal fees from Bristol-Myers Squibb, grants and non-financial support from Singulex, grants and non-financial support from Abbott, grants and non-financial support from Roche Diagnostics, and grants and non-financial support from Beckmann, outside the submitted work.

Ethics Approval: The study protocol and study documents were approved by the s Committee of Ulm University (No. 164/14).

Consent to Participate: Written informed consent was obtained from each subject upon entry into the study.

Consent for Publication: All patients have consented to their data being published. This is included in the informed consent.

Availability of Data and Material: Due to ethical restrictions regarding data protection issues and the study-specific consent text and procedure, the data cannot be made publicly available, but data are available to all interested researchers upon request.

\section{References}

1. Nüesch, E.; Dieppe, P.; Reichenbach, S.; Williams, S.; Iff, S.; Jüni, P. All cause and disease specific mortality in patients with knee or hip osteoarthritis: Population based cohort study. BMJ 2011, 342, d1165. [CrossRef] [PubMed]

2. Peters, M.J.L.; Symmons, D.P.M.; McCarey, D.; Dijkmans, B. a. C.; Nicola, P.; Kvien, T.K.; McInnes, I.B.; Haentzschel, H.; Gonzalez-Gay, M.A.; Provan, S.; et al. EULAR evidence-based recommendations for cardiovascular risk management in patients with rheumatoid arthritis and other forms of inflammatory arthritis. Ann. Rheum. Dis. 2010, 69, 325-331. [CrossRef] [PubMed]

3. Hall, A.J.; Stubbs, B.; Mamas, M.A.; Myint, P.K.; Smith, T.O. Association between osteoarthritis and cardiovascular disease: Systematic review and meta-analysis. Eur. J. Prev. Cardiol. 2016, 23, 938-946. [CrossRef]

4. Goff, D.C.; Lloyd-Jones, D.M.; Bennett, G.; Coady, S.; D’Agostino, R.B.; Gibbons, R.; Greenland, P.; Lackland, D.T.; Levy, D.; O'Donnell, C.J.; et al. 2013 ACC/AHA guideline on the assessment of cardiovascular risk: A report of the american college of cardiology/american heart association task force on practice guidelines. Circulation 2014, 129, S49-S73. [CrossRef] [PubMed] 
5. Hippisley-Cox, J.; Coupland, C.; Vinogradova, Y.; Robson, J.; Minhas, R.; Sheikh, A.; Brindle, P. Predicting cardiovascular risk in England and Wales: Prospective derivation and validation of QRISK2. BMJ 2008, 336, 1475-1482. [CrossRef] [PubMed]

6. D'Agostino, R.B.; Vasan, R.S.; Pencina, M.J.; Wolf, P.A.; Cobain, M.; Massaro, J.M.; Kannel, W.B. General cardiovascular risk profile for use in primary care: The framingham heart study. Circulation 2008, 117, 743-753. [CrossRef]

7. Conroy, R.M.; Pyörälä, K.; Fitzgerald, A.P.; Sans, S.; Menotti, A.; De Backer, G.; De Bacquer, D.; Ducimetière, P.; Jousilahti, P.; Keil, U.; et al. Estimation of ten-year risk of fatal cardiovascular disease in Europe: The score project. Eur. Heart J. 2003, 24, 987-1003. [CrossRef]

8. Ridker, P.M.; Paynter, N.P.; Rifai, N.; Gaziano, J.M.; Cook, N.R. C-reactive protein and parental history improve global cardiovascular risk prediction: The reynolds risk score for men. Circulation 2008, 118, 2243-2251. [CrossRef]

9. Arts, E.E.A.; Popa, C.; Den Broeder, A.A.; Semb, A.G.; Toms, T.; Kitas, G.D.; van Riel, P.L.; Fransen, J. Performance of four current risk algorithms in predicting cardiovascular events in patients with early rheumatoid arthritis. Ann. Rheum. Dis. 2015, 74, 668-674. [CrossRef]

10. Ozen, G.; Sunbul, M.; Atagunduz, P.; Direskeneli, H.; Tigen, K.; Inanc, N. The 2013 ACC/AHA 10-year atherosclerotic cardiovascular disease risk index is better than SCORE and QRisk II in rheumatoid arthritis: Is it enough? Rheumatology 2016, 55, 513-522. [CrossRef]

11. Solomon, D.H.; Greenberg, J.; Curtis, J.R.; Liu, M.; Farkouh, M.E.; Tsao, P.; Kremer, J.M.; Etzel, C.J. Derivation and internal validation of an expanded cardiovascular risk prediction score for rheumatoid arthritis: A consortium of rheumatology researchers of north america registry study. Arthritis Rheumatol. 2015, 67, 1995-2003. [CrossRef] [PubMed]

12. Agca, R.; Heslinga, S.C.; Rollefstad, S.; Heslinga, M.; McInnes, I.B.; Peters, M.J.L.; Kvien, T.K.; Dougados, M.; Radner, H.; Atzeni, F.; et al. Eular recommendations for cardiovascular disease risk management in patients with rheumatoid arthritis and other forms of inflammatory joint disorders: 2015/2016 update. Ann. Rheum. Dis. 2017, 76, 17-28. [CrossRef] [PubMed]

13. Crowson, C.S.; Gabriel, S.E.; Semb, A.G.; van Riel, P.L.C.M.; Karpouzas, G.; Dessein, P.H.; Hitchon, C.; Pascual-Ramos, V.; Kitas, G.D.; Douglas, K.; et al. Rheumatoid arthritis-specific cardiovascular risk scores are not superior to general risk scores: A validation analysis of patients from seven countries. Rheumatology 2017, 56, 1102-1110. [CrossRef] [PubMed]

14. Ahearn, J.; Shields, K.J.; Liu, C.-C.; Manzi, S. Cardiovascular disease biomarkers across autoimmune diseases. Clin. Immunol. 2015, 161, 59-63. [CrossRef] [PubMed]

15. Stürmer, T.; Brenner, H.; Koenig, W.; Günther, K.-P. Severity and extent of osteoarthritis and low grade systemic inflammation as assessed by high sensitivity $C$ reactive protein. Ann. Rheum. Dis. 2004, 63, $200-205$. [CrossRef] [PubMed]

16. Corre, J.; Hébraud, B.; Bourin, P. Concise review: Growth differentiation factor 15 in pathology: A clinical role? Stem Cells Transl. Med. 2013, 2, 946-952. [CrossRef] [PubMed]

17. Wollert, K.C.; Kempf, T.; Wallentin, L. Growth differentiation factor 15 as a biomarker in cardiovascular disease. Clin. Chem. 2017, 63, 140-151. [CrossRef] [PubMed]

18. Wang, J.; Wei, L.; Yang, X.; Zhong, J. Roles of growth differentiation factor 15 in atherosclerosis and coronary artery disease. J. Am. Heart Assoc. 2019, 8, e012826. [CrossRef]

19. Wallentin, L.; Hijazi, Z.; Andersson, U.; Alexander, J.H.; De Caterina, R.; Hanna, M.; Horowitz, J.D.; Hylek, E.M.; Lopes, R.D.; Asberg, S.; et al. Growth differentiation factor 15, a marker of oxidative stress and inflammation, for risk assessment in patients with atrial fibrillation: Insights from the Apixaban for reduction in stroke and other thromboembolic events in atrial fibrillation (Aristotle) trial. Circulation 2014, 130, 1847-1858. [CrossRef]

20. Hagström, E.; James, S.K.; Bertilsson, M.; Becker, R.C.; Himmelmann, A.; Husted, S.; Katus, H.A.; Steg, P.G.; Storey, R.F.; Siegbahn, A.; et al. Growth differentiation factor-15 level predicts major bleeding and cardiovascular events in patients with acute coronary syndromes: Results from the Plato study. Eur. Heart J. 2016, 37, 1325-1333. [CrossRef]

21. Vermeulen, B.; Schutte, A.E.; Gafane-Matemane, L.F.; Kruger, R. Growth differentiating factor-15 and its association with traditional cardiovascular risk factors: The African-Predict study. Nutr. Metab. Cardiovasc. Dis. 2020, 30, 925-931. [CrossRef] [PubMed] 
22. Wiklund, F.E.; Bennet, A.M.; Magnusson, P.K.E.; Eriksson, U.K.; Lindmark, F.; Wu, L.; Yaghoutyfam, N.; Marquis, C.P.; Stattin, P.; Pedersen, N.L.; et al. Macrophage inhibitory cytokine-1 (MIC-1/GDF15): A new marker of all-cause mortality. Aging Cell 2010, 9, 1057-1064. [CrossRef] [PubMed]

23. Daniels, L.B.; Clopton, P.; Laughlin, G.A.; Maisel, A.S.; Barrett-Connor, E. Growth-differentiation factor-15 is a robust, independent predictor of 11-year mortality risk in community-dwelling older adults: The rancho bernardo study. Circulation 2011, 123, 2101-2110. [CrossRef] [PubMed]

24. Wallentin, L.; Zethelius, B.; Berglund, L.; Eggers, K.M.; Lind, L.; Lindahl, B.; Wollert, K.C.; Siegbahn, A. GDF-15 for prognostication of cardiovascular and cancer morbidity and mortality in men. PLoS ONE 2013, 8, e78797. [CrossRef]

25. Rothenbacher, D.; Dallmeier, D.; Christow, H.; Koenig, W.; Denkinger, M.; Klenk, J. ActiFE study group Association of growth differentiation factor 15 with other key biomarkers, functional parameters and mortality in community-dwelling older adults. Age Ageing 2019, 48, 541-546. [CrossRef]

26. Wallentin, L.; Lindholm, D.; Siegbahn, A.; Wernroth, L.; Becker, R.C.; Cannon, C.P.; Cornel, J.H.; Himmelmann, A.; Giannitsis, E.; Harrington, R.A.; et al. Biomarkers in relation to the effects of ticagrelor in comparison with clopidogrel in non-ST-elevation acute coronary syndrome patients managed with or without in-hospital revascularization: A substudy from the prospective randomized platelet inhibition and patient outcomes (plato) trial. Circulation 2014, 129, 293-303. [CrossRef]

27. Hagström, E.; Held, C.; Stewart, R.A.H.; Aylward, P.E.; Budaj, A.; Cannon, C.P.; Koenig, W.; Krug-Gourley, S.; Mohler, E.R.; Steg, P.G.; et al. Growth differentiation factor 15 predicts all-cause morbidity and mortality in stable coronary heart disease. Clin. Chem. 2017, 63, 325-333. [CrossRef]

28. Wollert, K.C.; Kempf, T.; Peter, T.; Olofsson, S.; James, S.; Johnston, N.; Lindahl, B.; Horn-Wichmann, R.; Brabant, G.; Simoons, M.L.; et al. Prognostic value of growth-differentiation factor-15 in patients with non-ST-elevation acute coronary syndrome. Circulation 2007, 115, 962-971. [CrossRef]

29. Dallmeier, D.; Brenner, H.; Mons, U.; Rottbauer, W.; Koenig, W.; Rothenbacher, D. Growth differentiation factor 15, its 12-month relative change, and risk of cardiovascular events and total mortality in patients with stable coronary heart disease: 10-year follow-up of the karola study. Clin. Chem. 2016, 62, 982-992. [CrossRef]

30. Schaub, N.; Reichlin, T.; Twerenbold, R.; Reiter, M.; Steuer, S.; Bassetti, S.; Stelzig, C.; Wolf, C.; Winkler, K.; Haaf, P.; et al. Growth differentiation factor-15 in the early diagnosis and risk stratification of patients with acute chest pain. Clin. Chem. 2012, 58, 441-449. [CrossRef]

31. Wang, T.J.; Wollert, K.C.; Larson, M.G.; Coglianese, E.; McCabe, E.L.; Cheng, S.; Ho, J.E.; Fradley, M.G.; Ghorbani, A.; Xanthakis, V.; et al. Prognostic utility of novel biomarkers of cardiovascular stress: The Framingham Heart Study. Circulation 2012, 126, 1596-1604. [CrossRef] [PubMed]

32. Büchele, G.; Günther, K.P.; Brenner, H.; Puhl, W.; Stürmer, T.; Rothenbacher, D.; Brenner, R.E. Osteoarthritis-patterns, cardio-metabolic risk factors and risk of all-cause mortality: 20 years follow-up in patients after hip or knee replacement. Sci. Rep. 2018, 8, 5253. [CrossRef] [PubMed]

33. Pencina, M.J.; D'Agostino, R.B.; Steyerberg, E.W. Extensions of net reclassification improvement calculations to measure usefulness of new biomarkers. Stat. Med. 2011, 30, 11-21. [CrossRef]

34. Kerr, K.F.; Wang, Z.; Janes, H.; McClelland, R.L.; Psaty, B.M.; Pepe, M.S. Net reclassification indices for evaluating risk prediction instruments: A critical review. Epidemiology 2014, 25, 114-121. [CrossRef] [PubMed]

35. Cardoso, A.L.; Fernandes, A.; Aguilar-Pimentel, J.A.; de Angelis, M.H.; Guedes, J.R.; Brito, M.A.; Ortolano, S.; Pani, G.; Athanasopoulou, S.; Gonos, E.S.; et al. Towards frailty biomarkers: Candidates from genes and pathways regulated in aging and age-related diseases. Ageing Res. Rev. 2018, 47, 214-277. [CrossRef] [PubMed]

36. Ferrucci, L.; Fabbri, E. Inflammageing: Chronic inflammation in ageing, cardiovascular disease, and frailty. Nat. Rev. Cardiol. 2018, 15, 505-522. [CrossRef]

37. Tanaka, T.; Biancotto, A.; Moaddel, R.; Moore, A.Z.; Gonzalez-Freire, M.; Aon, M.A.; Candia, J.; Zhang, P.; Cheung, F.; Fantoni, G.; et al. Plasma proteomic signature of age in healthy humans. Aging Cell 2018, 17, e12799. [CrossRef]

38. Basisty, N.; Kale, A.; Jeon, O.H.; Kuehnemann, C.; Payne, T.; Rao, C.; Holtz, A.; Shah, S.; Sharma, V.; Ferrucci, L.; et al. A proteomic atlas of senescence-associated secretomes for aging biomarker development. PLoS Biol. 2020, 18, e3000599. [CrossRef] 
39. Doerstling, S.; Hedberg, P.; Öhrvik, J.; Leppert, J.; Henriksen, E. Growth differentiation factor 15 in a community-based sample: Age-dependent reference limits and prognostic impact. Ups. J. Med. Sci. 2018, 123, 86-93. [CrossRef]

40. Dallmeier, D.; Pencina, M.J.; Rajman, I.; Koenig, W.; Rothenbacher, D.; Brenner, H. Serial measurements of $\mathrm{N}$-terminal pro-brain natriuretic peptide in patients with coronary heart disease. PLOS ONE 2015, 10, e0117143. [CrossRef]

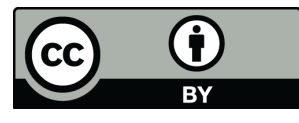

(C) 2020 by the authors. Licensee MDPI, Basel, Switzerland. This article is an open access article distributed under the terms and conditions of the Creative Commons Attribution (CC BY) license (http://creativecommons.org/licenses/by/4.0/). 